\title{
Tumor Extraction by Level Set Method using Thershold Algorithm
}

\author{
Visu Prateek Agarwal \\ Deptt. Of ECE \\ G.B.Pant Engineering College \\ Pauri Garhwal, Uttarakhand, India
}

\author{
Manoj Kumar \\ Deptt. Of ECE \\ G.B.Pant Engineering College \\ Pauri Garhwal, Uttarakhand, India
}

\begin{abstract}
The level set method may be used as a strongest pawn for segmentation of a tumor to achieve an accurate estimation of its volume. The level set method is a numeric technique for tracking interfaces and shapes. In this method, equation parameters are being set or speed function is being set. A threshold based method is introduced for tumor segmentation. In this paper, tumor segmentation and its extraction is achieved by a threshold based scheme and by utilizing a global threshold, the level set speed function is designed. This threshold based scheme provides better flexibility and it is updated through the whole process. Search based and adaptive bases threshold can be used here for better efficiency through segmentation. Tumor segmentation does not need any vast knowledge about the tumor and non-tumor density function. Depending upon the tumor shape and size, it may be implemented in an automatic or semi-automatic form. Here we use this algorithm for magnetic resonance images (MRI). We see that the performance can be evaluated accurately for quantitatively images. The results from this experiment provide better efficiency and high performance.
\end{abstract}

\section{INTRODUCTION}

Automated brain tumor segmentation from MRI images is really a very challenging job. It involves disciplines pathology covering, physics of MR images, perception of radiologists and on the basis of intensity and size, image is analyzed. There are many problems and issues with brain tumor segmentation. The size of brain tumor is different in different MR images and the shapes also have variation. Tumor can be located at any location having different intensities of images. For segmentation process, manual segmentation is quite difficult somewhere, that is why automated brain tumor segmentation method is more desirable. Maximum diameter of tumor is considered to be an indication of tumor size, and on account for 3D nature and irregularity in the shape of tumor it may not reflect a proper assessment of this tumor attribute $[1,2]$. Tumor size can be represented by tumor volume. This is how tumor volume can be estimated by tumor volume. Such types of methods schemes implicitly require the volume of tumor by extracting the tumor surface. By using 3D segmentation or via a pseudo-3D approach, extraction of surface can be achieved i.e. by using extracted 2D contours, reconstruction of 3Dsurface is done [3]. Due to higher accuracy of the former approach, to work on it is quite better. There are many methods or approaches used for estimating volume of tumor and extracting tumor. To measure the volume of tumor in MR images, fuzzy connectedness is a very useful and accurate method [4,5]. There are also some popular method for processing any medical image such as Markov random fields (MRFs) [6,7]. Utilization of support vector machine (SVM) classification method and brain tumor segmentation MRFs was evaluated by Lee et al. [8] and he claimed SVM based approach. Recently, Corso et al. [9] gave the extended graph-shifts algorithm for segmentation of image which exhibits many applications in tumor segmentation. Also, in a publication [10] the Bayesian generative model was made into the graph-based image segmentation and was applied to brain tumor segmentation. For segmentation of 3D objects, active surfaces/contours are other effective and very useful methods. Level set segmentation method has many advantages as it has a greater ability to tackle complex geometries and to handle changes in topology. The level set method provides shape driven method and level set method is bases upon speed function. It can shrink or grow to take the shape of any type of object whether it is very complex. Parameterization surface is not an issue with level set method [11]. Thus the level set method is very useful and it has high flexibility in modeling any shape and it is attractive and when we provide the initial zero level set, the whole segmentation process becomes automatic then. Level set method does not require any additional machinery. Level set method solves the problem of image segmentation which has minimum energy. The main theme for level set method is consisting of curves as a function which has higher dimensions that produces under some forces and it was firstly introduced by Osher and Sethian [12]. It provides efficient and accurate numerical techniques for analyzing and tracking curve evolution problems. Moreover, the contours of level set-based are independent parametrically and thus they are particularly appropriate for segmenting complex shapes. The evolution contour can naturally change its topology and result is got on less dependency on initialization as compared to active contour models. There are many approaches which have been proposed to address segmentation of MR images with level set method. Geodesic active contour, which was proposed in [13], has been extended to medical imagery in [14] and [15]. However, geodesic deformable contours shows there dependency on attributes of an image, for an example as gradient, that is why they are sensitive to noise. In [16], Zeng et al. applied a method for segmentation of three-dimensional (3-D) brain cortex which is done by using coupled level set surface propagation which is based on certain geometric parameters of thickness of cortex. In level set method user can tune and change the parameters according to his desire that is why this method is very useful. The user can also control the shape in a real time [17-19]. The drawback of this method is that this method provides user interaction and allows better segmentation only if user is totally familiar with the level set method. The main function in level set method is speed function. The design of speed function is the most important step. The speed function is defined based on results of various clustering algorithms ex. fuzzy classification [20-22]. Ho et al.[23]provides region competition in the exertion of the level set method, in which the speed function is adjusted through the difference between pre- and post-contrast enhanced magnetic resonance images. These algorithms also have some drawback that they depend upon accurate tumor and non tumor probability density functions estimation. It does not give accuracy sufficiently because of intensity differences in tumor. There are so many 
algorithms they are distinguished on the basis of the speed function. Some algorithms require user interaction while some rely on prior estimation of the density function of tumor. Thereby, a level set method is purposed for 3D brain tumor segmentation which employs density function is not required by a speed function and that is obtained by user interaction [24]. To form a speed function, global threshold is used. First of all, using level set initialization, the initial threshold is calculated and is then updated iteratively throughout the segmentation process. The variation of threshold declines while reaching tumor boundary because of the intensity difference between tumor and non tumor zone. The difficulty of our method undergo in the trade-off between the rate of convergence and segmentation accuracy. A high convergence rate is taken when the variation of threshold with respect to the iteration number is large. That is why accuracy is less in segmentation. A small variation in threshold can provide convergence whether of a reduced rate. This, however, may lead to low accuracy in segmentation or even destabilization of the algorithm. On the other hand, a small variation of the threshold can guarantee convergence, although at a reduced rate. We study here this trade-off and propose an appropriate calculation of threshold. Here we introduce a method that calculates a global threshold for segmentation of tumor, thereby it is unnecessary to estimate density of the tumor and non-tumor. Here we propose an algorithm for updating threshold iteratively.

\section{SEGMENTATION PROCESS}

For the process of segmentation, the image is divided into four regions: white matter (WM), grey matter (GM), cerebro spinal fluid (CSF) and background. For segmentation, fuzzy classification is done of voxels which is intensity based into background classes and tumor. Here tumor probability map is very useful. This map is used to guide speed of level set snake and propogation direction. It is also useful in deriving initialization automatically of snake. Image forces are balanced to cover a smooth tumor stable.

The image shows different aspects of tumor regions. First of all a seed point is selected in an image whether manually or automatically. Then on the basis of that seed point which is also known as an initial point, the region growing algorithms is applied and the pixels which have the same intensity value comes in a group and in the same manners, other pixels are also deformed in grouping. In this way segmentation process occurs. An example of an image of tumor is given below:
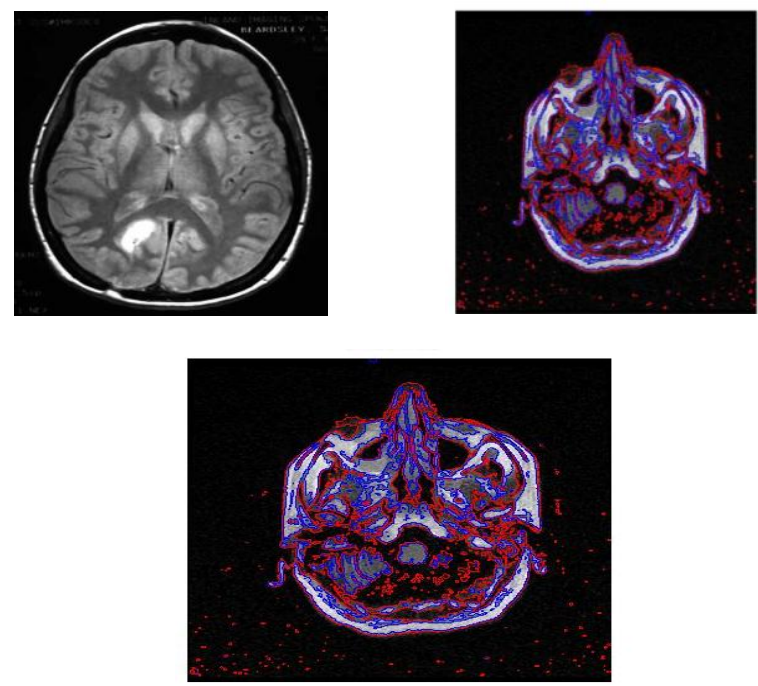

Fig 1. A) Original image of tumor. B) Segmented image. C) Segmented image with 99 iterations.

\section{THRESHOLD BASED}

\section{SEGMENTATION USING LEVEL SET}

Here we suppose that the tumor and adjacent non tumor histograms are overlapping slightly. We suppose that the region of background has lower mean intensity value than tumor region and there is no loss of generality. In this situation, a threshold exists between voxels of tumor and non tumor. Here we use an algorithm to find out proper threshold and a way to updating it on the basis of iterations. User performs the initial value of threshold which is based upon the level set initialization inside the region of tumor. After that a speed function is also performed. In it, knowledge of tumor and non tumor regions density functions is not required. To determine the speed function of level set is a challenging job. Image gradient is used by original formulation of speed function. When image is noisy or when the desire object's boundary is not distinct, the speed function does not work $[25,11]$. To improve the level set performance, regions information is integrated. We see that when tumor likelihood regions are higher, the growing speed increases. Estimation of threshold $\mathrm{T} 1$ process occurs by initializing TLS in tumor region. Reference slice has very large crosssectional area as compared to others slices. It must be notice that result will not be affected by the choice of reference slice.

In Fig. 2, we can choose an appropriate threshold from the shaded intensity ranges, which are where the final (converged) thresholds are very likely tolie. This range is expected to be wider when tumor and non tumor densities are also separated widely.

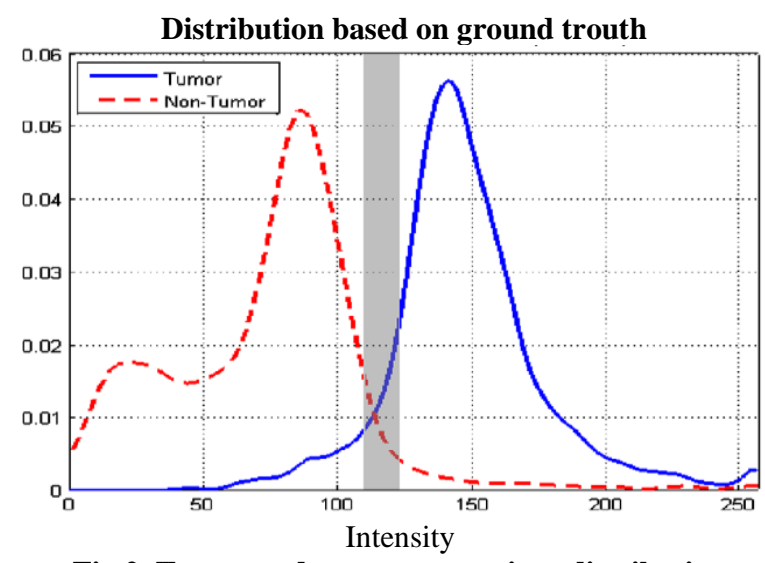

Fig 2. Tumor and non tumor regions distribution

TLS Algorithm:
1. Initialize the level set inside the tumor image.
2. Find out threshold
3. Compute speed function
4. Growing level set
5. Update the threshold
6. Reinitialize level set

\section{TUMOR EXTRACTION}

To extract the tumor, first of all we have to segment the image. After finding seed point and applying region growing algorithm, we can extract tumor on the basis of region. The intensity value of tumor region is different than background or other part of a tumor image. The pixel value is different in the tumor part as compared with other part of an image. Extracting tumor with level set method, we have to perform a task to find out speed function firstly and after that a value for threshold is provided. Then, the value above the threshold is taken out. This value is quite important for extracting tumor. 
Anexample of tumor image and segmented image and extracted tumor is given below:
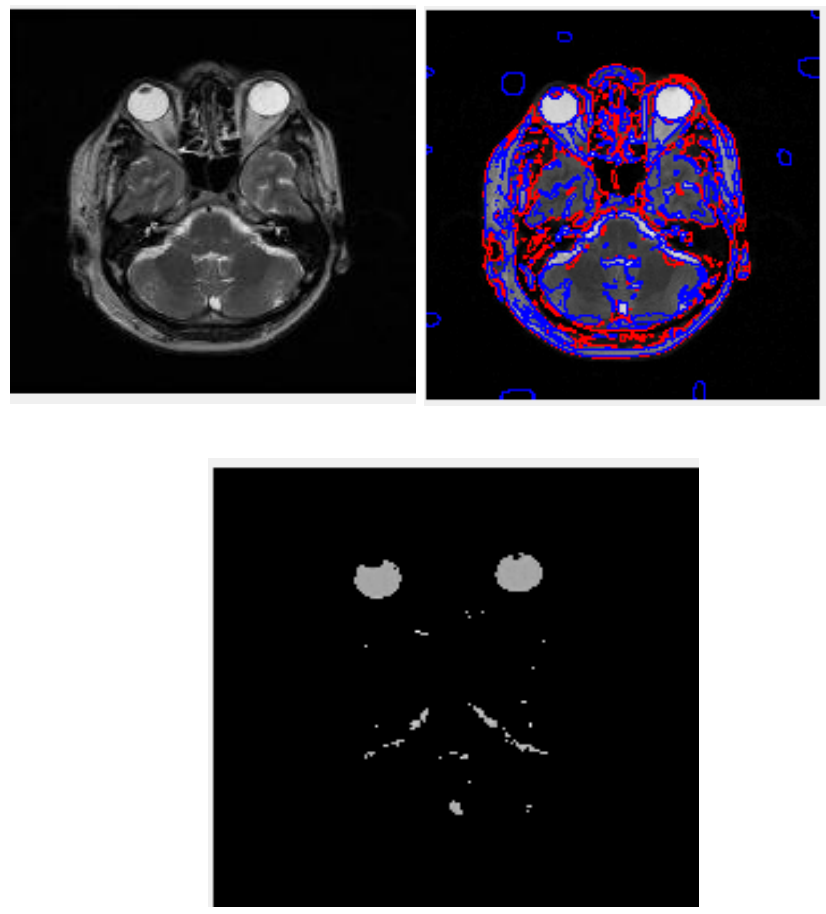

Fig 3. A) Original image of tumor. B) Segmented image with 99 iterations C) Extracted tumors

\section{CONCLUSION AND FUTURE WORK}

Here, in this paper we tumor extraction is done by a new method of level set method which is based on threshold algorithms. The densities of tumor region and non tumor regions are not required and main task is to find out the speed function. Level set method performance is better when the intensity difference level is different between tumor and non tumor regions. For convex case the efficiency and performance is better and for TLS performance is better where the level of intensity difference between the tumor and non-tumor regions is the case of concave, good initialization is necessary to obtain good segmentation. This method can also extract non-homogeneous tumors.

In this paper, a global threshold is achieved to perform tumor extraction. This TLS method is quite useful to find out global threshold and we can take some thresholds also except this. Threshold value is quite useful in finding the tumor. Where the global threshold value is not taken out then this TLS method will not work suitably. Except this some other abnormality detection reduces user interaction and thus the algorithm becomes automatic. In future work, author wants to try to reduce it and to work upon it.

\section{REFERENCES}

[1]. S. Saini, Radiologic measurement of tumor size in clinical trials: past, present, and future, American Journal of Roentgenol 176 (2) (2001) 333-334.

[2] A. Sorensen, S. Patel, C. Harmath, et al., Comparison of diameter and perimeter methods for tumor volume calculation, Journal of Clinical Oncology 19 (2) (2001) $551-557$

[3] J. Zhou, T. Lim, V. Chong, J. Huang, Segmentation and visualization of nasopharyngeal carcinoma using MRI,
Computers in Biology and Medicine 33 (5) (2003) 407424.

[4] G. Moonis, J. Liu, J. Udupa, D. Hackney, Estimation of tumor volume with fuzzy connectedness Segmentation of MR images, AJNR Am J Neuroradiol 23 (3) (2002) 356363.

[5] J. Liu, J. Udupa, D. Odhner, D. Hackney, G. Moonis, A system for brain tumor volume estimation via $\mathrm{mr}$ imaging and fuzzy connectedness, Computerized Medical Imaging and Graphics 29 (1) (2005) 21-34.

[6] K. Held, E. Kops, B. Krause, W. Wells, R. Kikinis, H. Muller-Gartner, Markov random field segmentation of brain $\mathrm{mr}$ images, IEEE Transactions on Medical Imaging 16 (1997) 878-886

[7] Y. Zhang, M. Brady, S. Smith, Segmentation of brain mr images through a hiddenmarkov random field model and the expectation-maximization algorithm, IEEE Transactions on Medical Imaging 20 (2001) 45-57.

[8] C. Lee, M. Schmidt, A. Murtha, A. Bistritz, J. Sander, R. Greiner, Segmenting brain tumor with conditional random fields and support vector machines, in: Proceedings of Workshop on Computer Vision for Biomedical Image Applications at International Conference on Computer Vision, 2005, pp. 469-478.

[9] J. Corso, A. Yuille, N. Sicotte, A. Toga, Detection and segmentation of pathological structures by the extended graph-shifts algorithm, in: Proceedings of Medical Image Computing and Computer Aided Intervention (MICCAI), vol. 1, 2007, pp. 985-994.

[10] J. Corso, E. Sharon, S. Dube, S. El-Saden, U. Sinha, A. Yuille, Efficient multilevel brain tumor segmentation with integrated bayesian model classification, IEEE Transactions on Medical Imaging 27 (5) (2008) 629-640

[11] J.A. Sethian, Level Set Methods and Fast Marching Methods: Evolving Interfaces in Geometry, Fluid Mechanics, Computer Vision, and Materials Science, second ed., Cambridge University Press, 1999.

[12]. S. Osher, and J. A. Sethian, "Fronts propagating with curvature-dependent speed: algorithms based on Harmilton-Jacobi formulations," Journal of Computational Physics, vol. 79, pp. 12-49, 1988

[13]. V. Caselles, R. Kimmel, and G. Sapiro, "On geodesic active contours," International Journal of Computer Vision, vol. 22, no. 1, pp. 61-79,1997

[14]. A. Yezzi, Jr., S. Kichenassamy, P. Olver, A. Kumar and A. Tannenbaum, "A Geometric Snake Model for Segmentation of Medical Imagery," IEEE Transactions on Medical Imaging, vol. 16, no.2, pp. 199-209, 1997

[15]. W. J. Niessen, B. M. ter Haar Romeny, and M. A. Viergever, "Geodesic Deformable Model for Medical Image Analysis," IEEE Transactions on Medical Imaging, vol. 17, no.4, PPpp. 634-641,1998

[16]. X. Zeng, L. H. Staib, R. T. Schultz, and J. S. Duncan, "Segmentation and Measurement of the Cortex from 3-D MR Images Using Coupled-surfaces," IEEE Transactions on Medical Imaging, vol. 18, pp. 927-937,1997

[17] M. Droske, B. Meyer, M. Rumpf, C. Schaller, An adaptive level set method for medical image 
segmentation, in: Proceedings of the Annual Symposium on Information Processing in Medical Imaging, 2001.

[18] S. Ho, H. Cody, G. Gerig, Snap: A software package for user-guided geodesic snake segmentation, Tech. rep., University of North Carolina, Chapel Hill (April 2003).

[20] Z. Zhou, J. You, P. Heng, D. Xia, Cardiacmr image segmentation and left ventricle surface reconstruction based on level set method, in: Studies in Health Technology and Informatics, 2005, pp. 629-632.

[21] Q. Su, K.-Y.K. Wong, G.S.K. Fung, A semi-automatic clustering-based level set method for segmentation of endocardium from msct images, in: Proceedings 29th Annual International Conference of the IEEE Engineering in Medicine and Biology Society, Lyon, France, 2007, pp. 6023-6026.

[22] H. Khotanlou, J. Atif, O. Colliot, I. Bloch, 3d brain tumor segmentation using fuzzy classification and deformable models, in: Int. Workshop on Fuzzy Logic and Applications, 2005, pp. 312-318.

[23] S. Ho, E. Bullitt, G. Gerig, Levelset evolution with region competition: automatic $3 \mathrm{~d}$ segmentation of brain tumors, in: Proc. of Int'l Conf. On Pattern Recognition, 2002, pp. 532-535.

[24] M. Prastawa, E. Bullitt, S. Ho, G. Gerig, A brain tumor segmentation framework based on outlier detection, Medical Image Analysis 8 (3) (2004) 275-283.

[25] S. Taheri, S. Ong, V. Chong, Threshold-based 3d tumor segmentation using level set (tsl), in: Proceedings of the Eighth IEEE Workshop on Applications of Computer Vision, 2007.

[26] R. Malladi, J.A. Sethian, B.C. Vemuri, Shapemodeling with front propagation: a level set approach, IEEE Trans. on Pattern Analysis and Machine Intelligence 17 (2) (1995) 158-175.

[27] D. Adalsteinsson, J. Sethian, A fast level set method for propagating interfaces, Journal of Computational Physics 118 (2) (1995) 269-277.

[24] B. Ostle, L. Malone, Statistics in Research: Basic Concepts and Techniques for Research Workers, 4th ed. Iowa State University Press, 1988.

[25] S.J. Osher, R.P. Fedkiw, Level Set Methods and Dynamic Implicit Surfaces,Springer,2003. 